\title{
A THEOREM ON TOPOLOGICAL MAPS
}

\author{
BY HEINZ KÖNIG
}

Communicated by Edwin Hewitt, July 13, 1962

Let $S$ be a topological space. We consider condition $\left({ }^{*}\right)$ : For each neighborhood $P$ of the diagonal in $S \times S$ there is a neighborhood $Q$ of the diagonal such that $Q \circ Q \subset P$ (neighborhoods are understood to be open sets). Condition $\left({ }^{* *}\right)$ means that $(*)$ is satisfied by each open subset of $S$ in the relative topology. Condition $\left(^{*}\right)$ is known to be an intermediate condition between full normality and normality of $S$ [1, problems 5.U and 6.L]. In particular, it is immediately seen that every metric space $S$ satisfies condition $\left({ }^{*}\right)$ and hence condition $\left({ }^{* *}\right)$.

We shall prove the following theorem. A special case (for metric spaces, in particular smooth manifolds in $R^{n}$ ) has recently been used [2] in the theory of partial differential equations of first order.

THEOREM. Let $S$ and $E$ be topological spaces satisfying condition (**) and $\phi$ a map of $S$ into E. Let $T$ be a subset of $S$. A ssume that

(i) $\phi$ is topological on $T$;

(ii) for each $x \in T$ there is a neighborhood $U(x)$ such that $\phi$ is topological on $U(x)$ and $\phi(U(x))$ is open.

Then there is a neighborhood $U$ of $T$ such that $\phi$ is topological on $U$ and $\phi(U)$ is open.

Proof. We put

$$
H=\bigcup_{x \in T} U(x) .
$$

Then $\phi$ is continuous on $H$. Furthermore, each open subset $A$ of $H$ has an open image $\phi(A)$, since we have

$$
A=\bigcup_{x \in T} A \cap U(x), \quad \phi(A)=\bigcup_{x \in T} \phi(A \cap U(x)),
$$

and $\phi(A \cap U(x))$ is open in view of (ii). Thus, if $\phi$ is one-to-one on an open subset $U$ of $H$, then $\phi$ is topological on $U$. We now construct in three steps a neighborhood $U \subset H$ of $T$ on which $\phi$ is one-to-one.

First step. The set

$$
P=\bigcup_{x \in T} U(x) \times U(x)
$$

is a neighborhood of the diagonal in $H \times H$. We take a neighborhood 
$Q$ of the diagonal such that $Q \circ Q \circ Q \subset P$, then for each $x \in T$ a neighborhood $V(x) \subset U(x)$ such that $V(x) \times V(x) \subset Q$.

Second step. For each $x \in T$, the set $V(x) \cap T$ is a neighborhood of $x$ in the relative topology of $T$. Hence, in view of (i), the set $\phi(V(x) \cap T)$ is a neighborhood of $\phi(x)$ in the relative topology of $\phi(T)$. This means that

$$
\phi(V(x) \cap T)=B \cap \phi(T)=B \cap \phi(V(x)) \cap \phi(T),
$$

where $B$ is a neighborhood of $\phi(x)$ in $E$. From (ii) we have $B \cap \phi(V(x))$ $=\phi(W(x))$, where $W(x) \subset V(x)$ is a neighborhood of $x$. It follows that $\phi(W(x)) \cap \phi(T)=\phi(V(x) \cap T)=\phi(W(x)) \cap \phi(V(x) \cap T)=\phi(W(x) \cap T)$, since $\phi$ is one-to-one on $V(x)$.

Third step. We put

$$
F=\bigcup_{x \in T} \phi(W(x))
$$

The set

$$
M=\bigcup_{x \in T} \phi(W(x)) \times \phi(W(x))
$$

is a neighborhood of the diagonal in $F \times F$. We take a neighborhood $N$ of the diagonal such that $N \circ N \subset M$, then for each $x \in T$ a neighborhood $R(x) \subset W(x)$ such that $\phi(R(x)) \times \phi(R(x)) \subset N$.

We now prove that $\phi$ is one-to-one on the neighborhood

$$
U=\bigcup_{x \in T} R(x) \subset H
$$

of $T$. Assume that

$$
\phi(p)=\phi(q) \text { for } p, q \in U: p \in R(x), q \in R(y) \quad \text { for } x, y \in T .
$$

Then we have $(\phi(x), \phi(p)),(\phi(q), \phi(y)) \in N$, hence $(\phi(x), \phi(y)) \in M$, or $\phi(x), \phi(y) \in \phi(W(a))$ for a certain $a \in T$. From the second step it follows that

$$
\phi(x), \phi(y) \in \phi(W(a)) \cap \phi(T)=\phi(W(a) \cap T),
$$

hence $x, y \in W(a)$, since $\phi$ is one-to-one on $T$. From this we have

$$
(p, x),(x, y),(y, q) \in Q,
$$

hence $(p, q) \in P$, or $p, q \in U(z)$ for a certain $z \in T$. This implies $p=q$, since $\phi$ is one-to-one on $U(z)$. The theorem is proved.

REMARK. We mention another version of the theorem which can be proved along the same lines. The conclusion of the theorem remains 
true if instead of condition $\left({ }^{* *}\right)$ for the spaces $S$ and $E$ one assumes that (i) $S$ is Hausdorff, (ii) each open subset of $E$ is fully normal in the relative topology, and (iii) one of the spaces $S$ and $E$ is locally connected.

\section{REFERENCES}

1. J. L. Kelley, General topology, Van Nostrand, New York, 1955.

2. H. König, Zur Theorie der partiellen Differentialgleichungen erster Ordnung, Arch. Math. (to appear).

The Technical University, Aachen 